\title{
Some Subordination and Superordination Results for Normalized Analytic Functions Defined by Convolution Structure Associated with Wanas Differential Operator
}

\author{
Abbas Kareem Wanas ${ }^{1}$ and Alb Lupas Alina ${ }^{2}$ \\ ${ }^{1}$ Department of Mathematics, College of Science, University of Al-Qadisiyah, Iraq \\ e-mail: abbas.kareem.w@qu.edu.iq \\ ${ }^{2}$ Department of Mathematics and Computer Science, University of Oradea, 1 Universitatii Street, \\ 410087 Oradea, Romania \\ e-mail: alblupas@gmail.com
}

\begin{abstract}
The purpose of this paper is to establish some subordination and superordination results involving Hadamard product for certain normalized analytic functions associated with Wanas differential operator defined in the open unit disk and obtain sandwich results. Our results extend corresponding previously known results.
\end{abstract}

\section{Introduction and Preliminaries}

Denote by $\mathcal{H}$ the class of analytic functions in the open unit disk $U=\{z \in \mathbb{C}:|z|<1\}$. For a positive integer $n$ and $a \in \mathbb{C}$, assume that $\mathcal{H}[a, n]$ is the subclass of $\mathcal{H}$ consisting of functions that have the form:

$$
f(z)=a+a_{n} z^{n}+a_{n+1} z^{n+1}+\cdots .
$$

Also, let $\mathcal{A}$ be the subclass of $\mathcal{H}$ consisting of functions of the form:

Received: March 8, 2020; Accepted: March 28, 2020

2010 Mathematics Subject Classification: 30C45, 30C80.

Keywords and phrases: analytic function, differential subordination, differential superordination, Hadamard product, Wanas differential operator.

Copyright (C) 2020 Abbas Kareem Wanas and Alb Lupas Alina. This is an open access article distributed under the Creative Commons Attribution License, which permits unrestricted use, distribution, and reproduction in any medium, provided the original work is properly cited. 


$$
f(z)=z+\sum_{n=2}^{\infty} a_{n} z^{n}
$$

For the functions $f \in \mathcal{A}$ given by (1.2) and $g \in \mathcal{A}$ defined by

$$
g(z)=z+\sum_{n=2}^{\infty} b_{n} z^{n}
$$

we define the Hadamard product (or convolution) of $f$ and $g$ by

$$
(f * g)(z)=z+\sum_{n=2}^{\infty} a_{n} b_{n} z^{n}=(g * f)(z) .
$$

With a view to recalling the principal of subordination between analytic functions, let $f, g \in \mathcal{H}$. The function $f$ is said to be subordinate to $g$, or $g$ is said to be superordinate to $f$, if there exists a Schwarz function $w$ analytic in $U$ with $w(0)=0$ and $|w(z)|<1(z \in U)$ such that $f(z)=g(w(z))$. In such a case we write $f \prec g$ or $f(z) \prec g(z)(z \in U)$. Furthermore, if $g$ is univalent in $U$, then we have the following equivalent (see [9]), $f \prec g \Leftrightarrow f(0)=g(0)$ and $f(U) \subset g(U)$.

Let $p, h \in \mathcal{H}$ and $\psi(r, s, t ; z): \mathbb{C}^{3} \times U \rightarrow \mathbb{C}$. If $p$ and $\psi\left(p(z), z p^{\prime}(z), z^{2} p^{\prime \prime}(z) ; z\right)$ are univalent functions in $U$ and if $p$ satisfies the second-order differential superordination:

$$
h(z) \prec \psi\left(p(z), z p^{\prime}(z), z^{2} p^{\prime \prime}(z) ; z\right),
$$

then $p$ is called a solution of the differential superordination (1.3). (If $f$ is subordinate to $g$, then $g$ is superordinate to $f$ ). An analytic function $q$ is called a subordinant of (1.3), if $q \prec p$ for all the functions $p$ satisfying (1.3). A univalent subordinant $\tilde{q}$ that satisfies $q \prec \tilde{q}$ for all the subordinants $q$ of (1.3) is called the best subordinant.

For $\alpha \in \mathbb{R}, \beta \geq 0$ with $\alpha+\beta>0, m, \eta \in \mathbb{N}_{0}=\mathbb{N} \cup\{0\}$ and $f \in \mathcal{A}$, the Wanas differential operator $W_{\alpha, \beta}^{k, \eta}: \mathcal{A} \rightarrow \mathcal{A}$ (see [16]) is defined by

$$
W_{\alpha, \beta}^{k, \eta} f(z)=z+\sum_{n=2}^{\infty}\left[\sum_{m=1}^{k}\left(\begin{array}{l}
k \\
m
\end{array}\right)(-1)^{m+1}\left(\frac{\alpha^{m}+n \beta^{m}}{\alpha^{m}+\beta^{m}}\right)\right]^{\eta} a_{n} z^{n} .
$$


It is easily verified from (1.2) that

$$
\begin{aligned}
z\left(W_{\alpha, \beta}^{k, \eta} f(z)\right)^{\prime}= & {\left[\sum_{m=1}^{k}\left(\begin{array}{l}
k \\
m
\end{array}\right)(-1)^{m+1}\left(\left(\frac{\alpha}{\beta}\right)^{m}+1\right)\right] W_{\alpha, \beta}^{k, \eta+1} f(z) } \\
& -\left[\sum_{m=1}^{k}\left(\begin{array}{l}
k \\
m
\end{array}\right)(-1)^{m+1}\left(\frac{\alpha}{\beta}\right)^{m}\right] W_{\alpha, \beta}^{k, \eta} f(z) .
\end{aligned}
$$

Special cases of this operator can be found in $[1,2,4,6,7,8,11,13,14,15]$. For more details see [19].

Very recently, Rahrovi [10], Attiya and Yassen [3], Seoudy [12] and Wanas and Lupas [18] have studied differential subordinations and superordinations for different conditions of analytic functions.

The main object of the present paper is to find sufficient condition for certain normalized analytic functions $f$ in $U$ such that $(f * \Psi)(z) \neq 0$ and $f$ to satisfy

$$
q_{1}(z) \prec \frac{W_{\alpha, \beta}^{k, \eta+1}(f * \Phi)(z)}{W_{\alpha, \beta}^{k, \eta}(f * \Psi)(z)} \prec q_{2}(z),
$$

where $q_{1}$ and $q_{2}$ are given univalent functions in $U$ and $\Phi(z)=z+\sum_{n=2}^{\infty} t_{n} z^{n}$, $\Psi(z)=z+\sum_{n=2}^{\infty} \delta_{n} z^{n}$ are analytic functions in $U$ with $t_{n} \geq 0, \delta_{n} \geq 0$ and $t_{n} \geq \delta_{n}$. Also, we obtain the number of results as their special cases.

To establish our main results, we need the following definition and lemmas:

Definition 1.1 [9]. Denote by $Q$ the set of all functions $f$ that are analytic and injective on $\bar{U} \backslash E(f)$, where

$$
E(f)=\left\{\zeta \in \partial U: \lim _{z \rightarrow \zeta} f(z)=\infty\right\}
$$

and are such that $f^{\prime}(\zeta) \neq 0$ for $\zeta \in \partial U \backslash E(f)$. 
Lemma 1.1 [9]. Let $q$ be univalent in the unit disk $U$ and let $\theta$ and $\phi$ be analytic in a domain D containing $q(U)$ with $\phi(w) \neq 0$ when $w \in q(U)$. Set $Q(z)=z q^{\prime}(z) \phi(q(z))$ and $h(z)=\theta(q(z))+Q(z)$. Suppose that

(1) $Q(z)$ is starlike univalent in $U$,

(2) $\operatorname{Re}\left\{\frac{z h^{\prime}(z)}{Q(z)}\right\}>0$ for $z \in U$.

If

$$
\theta(p(z))+z p^{\prime}(z) \phi(p(z)) \prec \theta(q(z))+z q^{\prime}(z) \phi(q(z)),
$$

then $p \prec q$ and $q$ is the best dominant of (1.6).

Lemma 1.2 [5]. Let $q$ be convex univalent in the unit disk $U$ and let $\theta$ and $\phi$ be analytic in a domain D containing $q(U)$. Suppose that

(1) $\operatorname{Re}\left\{\frac{\theta^{\prime}(q(z))}{\phi(q(z))}\right\}>0$ for $z \in U$,

(2) $Q(z)=z q^{\prime}(z) \phi(q(z))$ is starlike univalent in $U$.

If $p \in \mathcal{H}[q(0), 1] \cap Q$, with $p(U) \subset D, \theta(p(z))+z p^{\prime}(z) \phi(p(z))$ is univalent in $U$ and

$$
\theta(q(z))+z q^{\prime}(z) \phi(q(z)) \prec \theta(p(z))+z p^{\prime}(z) \phi(p(z)),
$$

then $q \prec p$ and $q$ is the best subordinant of (1.7).

\section{Main Results}

Theorem 2.1. Let $\Phi, \Psi \in \mathcal{A}$ and $q$ be univalent in $U$ with $q(z) \neq 0, q(0)=1$ and assume that

$$
\operatorname{Re}\left\{1+\frac{\lambda_{2}(\gamma-\sigma)}{\lambda_{3} \sigma}+\frac{\lambda_{1} \gamma}{\lambda_{3} \sigma} q(z)+\left(\frac{\gamma}{\sigma}-2\right) \frac{z q^{\prime}(z)}{q(z)}+\frac{z q^{\prime \prime}(z)}{q^{\prime}(z)}\right\}>0
$$


where $\lambda_{1}, \lambda_{2}, \gamma \in \mathbb{C}, \lambda_{3}, \sigma \in \mathbb{C} \backslash\{0\}$. Suppose that $z(q(z))_{\sigma}^{\frac{\gamma}{\sigma}}-2 q^{\prime}(z)$ is starlike univalent in $U$. If $f \in \mathcal{A}, \frac{W_{\alpha, \beta}^{k, \eta+1}(f * \Phi)(z)}{W_{\alpha, \beta}^{k, \eta}(f * \Psi)(z)} \neq 0, z \in U$, satisfies the differential subordination

$\Upsilon_{1}\left(f, \Phi, \Psi, \lambda_{1}, \lambda_{2}, \lambda_{3}, \gamma, \sigma, \alpha, \beta, k, \eta ; z\right) \prec(q(z))^{\gamma}\left(\lambda_{1}+\frac{\lambda_{2}}{q(z)}+\lambda_{3} \frac{z q^{\prime}(z)}{(q(z))^{2}}\right)^{\sigma}$,

where

$$
\begin{aligned}
& \Upsilon_{1}\left(f, \Phi, \Psi, \lambda_{1}, \lambda_{2}, \lambda_{3}, \gamma, \sigma, \alpha, \beta, k, \eta ; z\right) \\
& =\left(\frac{W_{\alpha, \beta}^{k, \eta+1}(f * \Phi)(z)}{W_{\alpha, \beta}^{k, \eta}(f * \Psi)(z)}\right)^{\gamma} \\
& \times\left(\lambda_{1}+\lambda_{2} \frac{W_{\alpha, \beta}^{k, \eta}(f * \Psi)(z)}{W_{\alpha, \beta}^{k, \eta+1}(f * \Phi)(z)}+\lambda_{3} \sum_{m=1}^{k}\left(\begin{array}{c}
k \\
m
\end{array}\right)(-1)^{m+1}\left(\left(\frac{\alpha}{\beta}\right)^{m}+1\right) \frac{W_{\alpha, \beta}^{k, \eta}(f * \Psi)(z)}{W_{\alpha, \beta}^{k, \eta+1}(f * \Phi)(z)}\right. \\
& \left.\times\left(\frac{W_{\alpha, \beta}^{k, \eta+2}(f * \Phi)(z)}{W_{\alpha, \beta}^{k, \eta+1}(f * \Phi)(z)}-\frac{W_{\alpha, \beta}^{k, \eta+1}(f * \Psi)(z)}{W_{\alpha, \beta}^{k, \eta}(f * \Psi)(z)}\right)\right)^{\sigma},
\end{aligned}
$$

then

$$
\frac{W_{\alpha, \beta}^{k, \eta+1}(f * \Phi)(z)}{W_{\alpha, \beta}^{k, \eta}(f * \Psi)(z)} \prec q(z)
$$

and $q$ is the best dominant.

Proof. Define the function $p$ by

$$
p(z)=\frac{W_{\alpha, \beta}^{k, \eta+1}(f * \Phi)(z)}{W_{\alpha, \beta}^{k, \eta}(f * \Psi)(z)}, \quad z \in U
$$


Differentiating (2.4) with respect to $z$ and using (1.5), we obtain

$$
\begin{aligned}
& (p(z))^{\gamma}\left(\lambda_{1}+\frac{\lambda_{2}}{p(z)}+\lambda_{3} \frac{z p^{\prime}(z)}{(p(z))^{2}}\right)^{\sigma} \\
= & \left(\frac{W_{\alpha, \beta}^{k, \eta+1}(f * \Phi)(z)}{W_{\alpha, \beta}^{k, \eta}(f * \Psi)(z)}\right)^{\gamma}\left(\lambda_{1}+\lambda_{2} \frac{W_{\alpha, \beta}^{k, \eta}(f * \Psi)(z)}{W_{\alpha, \beta}^{k, \eta+1}(f * \Phi)(z)}\right. \\
+ & \left.\frac{\lambda_{3}(\alpha+\beta)}{\beta} \frac{W_{\alpha, \beta}^{k, \eta}(f * \Psi)(z)}{W_{\alpha, \beta}^{k, \eta+1}(f * \Phi)(z)}\left(\frac{W_{\alpha, \beta}^{k, \eta+2}(f * \Phi)(z)}{W_{\alpha, \beta}^{k, \eta+1}(f * \Phi)(z)}-\frac{W_{\alpha, \beta}^{k, \eta+1}(f * \Psi)(z)}{W_{\alpha, \beta}^{k, \eta}(f * \Psi)(z)}\right)\right)^{\sigma} .
\end{aligned}
$$

In view of (2.2) and (2.5), we have

$$
(p(z))^{\gamma}\left(\lambda_{1}+\frac{\lambda_{2}}{p(z)}+\lambda_{3} \frac{z p^{\prime}(z)}{(p(z))^{2}}\right)^{\sigma} \prec(q(z))^{\gamma}\left(\lambda_{1}+\frac{\lambda_{2}}{q(z)}+\lambda_{3} \frac{z q^{\prime}(z)}{(q(z))^{2}}\right)^{\sigma}
$$

This equivalently to

$$
(p(z)) \frac{\gamma}{\sigma}\left(\lambda_{1}+\frac{\lambda_{2}}{p(z)}+\lambda_{3} \frac{z p^{\prime}(z)}{(p(z))^{2}}\right) \prec(q(z)) \frac{\gamma}{\sigma}\left(\lambda_{1}+\frac{\lambda_{2}}{q(z)}+\lambda_{3} \frac{z q^{\prime}(z)}{(q(z))^{2}}\right) .
$$

By setting

$$
\theta(w)=\left(\lambda_{1} w+\lambda_{2}\right) w^{\frac{\gamma}{\sigma}-1} \text { and } \phi(w)=\lambda_{3} w^{\frac{\gamma}{\sigma}-2} \text {, }
$$

it can be easily observed that $\theta(w)$ and $\phi(w)$ are analytic in $\mathbb{C} \backslash\{0\}$ and that $\phi(w) \neq 0, w \in \mathbb{C} \backslash\{0\}$. Also, we get

$$
Q(z)=z q^{\prime}(z) \phi(q(z))=\lambda_{3} z(q(z)) \frac{\gamma}{\sigma}-2 q^{\prime}(z)
$$

and

$$
h(z)=\theta(q(z))+Q(z)=(q(z)) \frac{\gamma}{\sigma}\left(\lambda_{1}+\frac{\lambda_{2}}{q(z)}+\lambda_{3} \frac{z q^{\prime}(z)}{(q(z))^{2}}\right) .
$$


It is clear that $Q(z)$ is starlike univalent in $U$,

$$
\operatorname{Re}\left\{\frac{z h^{\prime}(z)}{Q(z)}\right\}=\operatorname{Re}\left\{1+\frac{\lambda_{2}(\gamma-\sigma)}{\lambda_{3} \sigma}+\frac{\lambda_{1} \gamma}{\lambda_{3} \sigma} q(z)+\left(\frac{\gamma}{\sigma}-2\right) \frac{z q^{\prime}(z)}{q(z)}+\frac{z q^{\prime \prime}(z)}{q^{\prime}(z)}\right\} .
$$

From (2.1) and (2.6), we have

$$
\operatorname{Re}\left\{\frac{z h^{\prime}(z)}{Q(z)}\right\}>0
$$

Therefore by an application of Lemma 1.1, we get $p(z) \prec q(z)$. By using (2.4), we obtain the desired result.

Remark 2.1. By taking $k=1$ in Theorem 2.1, we obtain the results for the operator $I_{\alpha, \beta}^{\eta}$ which was obtained recently by Wanas and Joudah [17, Theorem 3.1].

By fixing $\Phi(z)=\Psi(z)=\frac{z}{1-z}$ in Theorem 2.1, we obtain the following corollary:

Corollary 2.1. Let $q$ be univalent in $U$ with $q(z) \neq 0, q(0)=1$ and assume that (2.1) holds true. Suppose that $z(q(z))_{\sigma}^{\frac{\gamma}{\sigma}-2} q^{\prime}(z)$ is starlike univalent in $U$. If $f \in \mathcal{A}$, $\frac{W_{\alpha, \beta}^{k, \eta+1} f(z)}{W_{\alpha, \beta}^{k, \eta} f(z)} \neq 0, z \in U$, satisfies the differential subordination

$$
\Upsilon_{2}\left(f, \lambda_{1}, \lambda_{2}, \lambda_{3}, \gamma, \sigma, \alpha, \beta, k, \eta ; z\right) \prec(q(z))^{\gamma}\left(\lambda_{1}+\frac{\lambda_{2}}{q(z)}+\lambda_{3} \frac{z q^{\prime}(z)}{(q(z))^{2}}\right)^{\sigma},
$$

where

$$
\begin{aligned}
& \Upsilon_{2}\left(f, \lambda_{1}, \lambda_{2}, \lambda_{3}, \gamma, \sigma, \alpha, \beta, k, \eta ; z\right) \\
= & \left(\frac{W_{\alpha, \beta}^{k, \eta+1} f(z)}{W_{\alpha, \beta}^{k, \eta} f(z)}\right)^{\gamma}\left(\lambda_{1}+\lambda_{2} \frac{W_{\alpha, \beta}^{k, \eta} f(z)}{W_{\alpha, \beta}^{k, \eta+1} f(z)}+\lambda_{3} \sum_{m=1}^{k}\left(\begin{array}{c}
k \\
m
\end{array}\right)(-1)^{m+1}\left(\left(\frac{\alpha}{\beta}\right)^{m}+1\right)\right. \\
& \left.\times \frac{W_{\alpha, \beta}^{k, \eta} f(z)}{W_{\alpha, \beta}^{k, \eta+1} f(z)}\left(\frac{W_{\alpha, \beta}^{k, \eta+2} f(z)}{W_{\alpha, \beta}^{k, \eta+1} f(z)}-\frac{W_{\alpha, \beta}^{k, \eta+1} f(z)}{W_{\alpha, \beta}^{k, \eta} f(z)}\right)\right)^{\sigma},
\end{aligned}
$$


then

$$
\frac{W_{\alpha, \beta}^{k, \eta+1} f(z)}{W_{\alpha, \beta}^{k, \eta} f(z)} \prec q(z)
$$

and $q$ is the best dominant.

Theorem 2.2. Let $\Phi, \Psi \in \mathcal{A}$ and $q$ be convex univalent in $U$ with $q(z) \neq 0$, $q(0)=1$ and assume that

$$
\operatorname{Re}\left\{\frac{\lambda_{2}(\gamma-\sigma)}{\lambda_{3} \sigma}+\frac{\lambda_{1} \gamma}{\lambda_{3} \sigma} q(z)\right\}>0
$$

where $\lambda_{1}, \lambda_{2}, \gamma \in \mathbb{C}, \lambda_{3}, \sigma \in \mathbb{C} \backslash\{0\}$. Suppose that $z\left(q(z) \frac{\gamma^{\frac{\gamma}{\sigma}}-2}{q^{\prime}(z)}\right.$ is starlike univalent in $\quad U . \quad$ Let $\quad f \in \mathcal{A}, \quad \frac{W_{\alpha, \beta}^{k, \eta+1}(f * \Phi)(z)}{W_{\alpha, \beta}^{k, \eta}(f * \Psi)(z)} \in \mathcal{H}[q(0), 1] \cap Q \quad$ with $\frac{W_{\alpha, \beta}^{k, \eta+1}(f * \Phi)(z)}{W_{\alpha, \beta}^{k, \eta}(f * \Psi)(z)} \neq 0, \quad z \in U$ and $\Upsilon_{1}\left(f, \Phi, \Psi, \lambda_{1}, \lambda_{2}, \lambda_{3}, \gamma, \sigma, \alpha, \beta, k, \eta ; z\right)$ be univalent in $U$, where $\Upsilon_{1}\left(f, \Phi, \Psi, \lambda_{1}, \lambda_{2}, \lambda_{3}, \gamma, \sigma, \alpha, \beta, k, \eta ; z\right)$ is given by (2.3). If

$$
\begin{aligned}
& (q(z))^{\gamma}\left(\lambda_{1}+\frac{\lambda_{2}}{q(z)}+\lambda_{3} \frac{z q^{\prime}(z)}{(q(z))^{2}}\right)^{\sigma} \\
\prec & \Upsilon_{1}\left(f, \Phi, \Psi, \lambda_{1}, \lambda_{2}, \lambda_{3}, \gamma, \sigma, \alpha, \beta, k, \eta ; z\right),
\end{aligned}
$$

then

$$
q(z) \prec \frac{W_{\alpha, \beta}^{k, \eta+1}(f * \Phi)(z)}{W_{\alpha, \beta}^{k, \eta}(f * \Psi)(z)}
$$

and $q$ is the best subordinant.

Proof. Let the function $p$ be defined by (2.4). After simple computation and making use of (1.5), the superordination (2.9) becomes

$$
(q(z))^{\gamma}\left(\lambda_{1}+\frac{\lambda_{2}}{q(z)}+\lambda_{3} \frac{z q^{\prime}(z)}{(q(z))^{2}}\right)^{\sigma} \prec(p(z))^{\gamma}\left(\lambda_{1}+\frac{\lambda_{2}}{p(z)}+\lambda_{3} \frac{z p^{\prime}(z)}{(p(z))^{2}}\right)^{\sigma} .
$$


This equivalently to

$$
(q(z)) \frac{\gamma}{\sigma}\left(\lambda_{1}+\frac{\lambda_{2}}{q(z)}+\lambda_{3} \frac{z q^{\prime}(z)}{(q(z))^{2}}\right) \prec(p(z)) \frac{\gamma}{\sigma}\left(\lambda_{1}+\frac{\lambda_{2}}{p(z)}+\lambda_{3} \frac{z p^{\prime}(z)}{(p(z))^{2}}\right) .
$$

By setting

$$
\theta(w)=\left(\lambda_{1} w+\lambda_{2}\right) w^{\frac{\gamma}{\sigma}-1} \text { and } \phi(w)=\lambda_{3} w^{\frac{\gamma}{\sigma}-2}
$$

it is easily observed that $\theta(w)$ and $\phi(w)$ are analytic in $\mathbb{C} \backslash\{0\}$ and that $\phi(w) \neq 0, w \in \mathbb{C} \backslash\{0\}$. Also, we get

$$
Q(z)=z q^{\prime}(z) \phi(q(z))=\lambda_{3} z(q(z))_{\sigma}^{\frac{\gamma}{\sigma}}-2 q^{\prime}(z) .
$$

It is clear that $Q(z)$ is starlike univalent in $U$,

$$
\operatorname{Re}\left\{\frac{\theta^{\prime}(q(z))}{\phi(q(z))}\right\}=\operatorname{Re}\left\{\frac{\lambda_{2}(\gamma-\sigma)}{\lambda_{3} \sigma}+\frac{\lambda_{1} \gamma}{\lambda_{3} \sigma} q(z)\right\} .
$$

From (2.8) and (2.10), we have

$$
\operatorname{Re}\left\{\frac{\theta^{\prime}(q(z))}{\phi(q(z))}\right\}>0
$$

Therefore by an application of Lemma 1.2, we get $q(z) \prec p(z)$. By using (2.4), we obtain the desired result.

Remark 2.2. By taking $k=1$ in Theorem 2.2, we obtain the results for the operator $I_{\alpha, \beta}^{\eta}$ which was obtained recently by Wanas and Joudah [17, Theorem 4.1].

By fixing $\Phi(z)=\Psi(z)=\frac{z}{1-z}$ in Theorem 4.1, we obtain the following corollary:

Corollary 2.2. Let $q$ be convex univalent in $U$ with $q(z) \neq 0, q(0)=1$ and assume that (2.8) holds true. Suppose that $z(q(z))_{\sigma}^{\frac{\gamma}{\sigma}}-2 q^{\prime}(z)$ is starlike univalent in $U$. Let $f \in \mathcal{A}, \frac{W_{\alpha, \beta}^{k, \eta+1} f(z)}{W_{\alpha, \beta}^{k, \eta} f(z)} \in \mathcal{H}[q(0), 1] \cap Q$ with $\frac{W_{\alpha, \beta}^{k, \eta+1} f(z)}{W_{\alpha, \beta}^{k, \eta} f(z)} \neq 0, z \in U$, and $\Upsilon_{2}\left(f, \lambda_{1}\right.$, 
$\left.\lambda_{2}, \lambda_{3}, \gamma, \sigma, \alpha, \beta, k, \eta ; z\right)$ be univalent in $U$, where $\Upsilon_{2}\left(f, \lambda_{1}, \lambda_{2}, \lambda_{3}, \gamma, \sigma, \alpha, \beta\right.$, $k, \eta$; $z$ ) is given by (2.7). If

$$
(q(z))^{\gamma}\left(\lambda_{1}+\frac{\lambda_{2}}{q(z)}+\lambda_{3} \frac{z q^{\prime}(z)}{(q(z))^{2}}\right)^{\sigma} \prec \Upsilon_{2}\left(f, \lambda_{1}, \lambda_{2}, \lambda_{3}, \gamma, \sigma, \alpha, \beta, k, \eta ; z\right),
$$

then

$$
q(z) \prec \frac{W_{\alpha, \beta}^{k, \eta+1} f(z)}{W_{\alpha, \beta}^{k, \eta} f(z)}
$$

and $q$ is the best subordinant.

Concluding the results of differential subordination and superordination, we arrive at the following "sandwich results".

Theorem 2.3. Let $\Phi, \Psi \in \mathcal{A}$. Let $q_{1}$ and $q_{2}$ be convex univalent in $U$ with $q_{1}(0)=q_{2}(0)=1$. Suppose $q_{2}$ satisfies (2.1) and $q_{1}$ satisfies (2.8). Let $f \in \mathcal{A}$, $\frac{W_{\alpha, \beta}^{k, \eta+1}(f * \Phi)(z)}{W_{\alpha, \beta}^{k, \eta}(f * \Psi)(z)} \in \mathcal{H}[1,1] \cap Q$ with $\frac{W_{\alpha, \beta}^{k, \eta+1}(f * \Phi)(z)}{W_{\alpha, \beta}^{k, \eta}(f * \Psi)(z)} \neq 0, z \in U$ and $\Upsilon_{1}(f, \Phi$, $\left.\Psi, \lambda_{1}, \lambda_{2}, \lambda_{3}, \gamma, \sigma, \alpha, \beta, k, \eta ; z\right)$ be univalent in $U$, where $\Upsilon_{1}\left(f, \Phi, \Psi, \lambda_{1}, \lambda_{2}, \lambda_{3}\right.$, $\gamma, \sigma, \alpha, \beta, k, \eta$; z) is given by (2.3). If

$$
\begin{aligned}
\left(q_{1}(z)\right)^{\gamma}\left(\lambda_{1}+\frac{\lambda_{2}}{q_{1}(z)}+\lambda_{3} \frac{z q_{1}^{\prime}(z)}{\left(q_{1}(z)\right)^{2}}\right)^{\sigma} & \prec \Upsilon_{1}\left(f, \Phi, \Psi, \lambda_{1}, \lambda_{2}, \lambda_{3}, \gamma, \sigma, \alpha, \beta, k, \eta ; z\right) \\
& \prec\left(q_{2}(z)\right)^{\gamma}\left(\lambda_{1}+\frac{\lambda_{2}}{q_{2}(z)}+\lambda_{3} \frac{z q_{2}^{\prime}(z)}{\left(q_{2}(z)\right)^{2}}\right)^{\sigma},
\end{aligned}
$$

then

$$
q_{1}(z) \prec \frac{W_{\alpha, \beta}^{k, \eta+1}(f * \Phi)(z)}{W_{\alpha, \beta}^{k, \eta}(f * \Psi)(z)} \prec q_{2}(z)
$$

and $q_{1}$ and $q_{2}$ are, respectively, the best subordinant and the best dominant. 
Remark 2.3. By taking $k=1$ in Theorem 2.3, we obtain the results for the operator $I_{\alpha, \beta}^{\eta}$ which was obtained recently by Wanas and Joudah [17, Theorem 5.1].

By making use of Corollaries 2.1 and 2.2, we obtain the following corollary:

Corollary 2.3. Let $q_{1}$ and $q_{2}$ be convex univalent in $U$ with $q_{1}(0)=q_{2}(0)=1$.

Suppose $q_{2}$ satisfies (2.1) and $q_{1}$ satisfies (2.8). Let $f \in \mathcal{A}, \frac{W_{\alpha, \beta}^{k, \eta+1} f(z)}{W_{\alpha, \beta}^{k, \eta} f(z)} \in \mathcal{H}[1,1] \cap Q$ with $\frac{W_{\alpha, \beta}^{k, \eta+1} f(z)}{W_{\alpha, \beta}^{k, \eta} f(z)} \neq 0, \quad z \in U$ and $\Upsilon_{2}\left(f, \lambda_{1}, \lambda_{2}, \lambda_{3}, \gamma, \sigma, \alpha, \beta, k, \eta ; z\right)$ be univalent in $U$, where $\Upsilon_{2}\left(f, \lambda_{1}, \lambda_{2}, \lambda_{3}, \gamma, \sigma, \alpha, \beta, k, \eta ; z\right)$ is given by (2.7). If

$$
\begin{aligned}
\left(q_{1}(z)\right)^{\gamma}\left(\lambda_{1}+\frac{\lambda_{2}}{q_{1}(z)}+\lambda_{3} \frac{z q_{1}^{\prime}(z)}{\left(q_{1}(z)\right)^{2}}\right)^{\sigma} & \prec \Upsilon_{2}\left(f, \lambda_{1}, \lambda_{2}, \lambda_{3}, \gamma, \sigma, \alpha, \beta, k, \eta ; z\right) \\
& \prec\left(q_{2}(z)\right)^{\gamma}\left(\lambda_{1}+\frac{\lambda_{2}}{q_{2}(z)}+\lambda_{3} \frac{z q_{2}^{\prime}(z)}{\left(q_{2}(z)\right)^{2}}\right)^{\sigma},
\end{aligned}
$$

then

$$
q_{1}(z) \prec \frac{W_{\alpha, \beta}^{k, \eta+1} f(z)}{W_{\alpha, \beta}^{k, \eta} f(z)} \prec q_{2}(z)
$$

and $q_{1}$ and $q_{2}$ are, respectively, the best subordinant and the best dominant.

\section{References}

[1] J. W. Alexander, Functions which map the interior of the unit circle upon simple region, Ann. of Math. 17(1) (1915), 12-22. https://doi.org/10.2307/2007212

[2] F. M. Al-Oboudi, On univalent functions defined by a generalized Salagean operator, Int. J. Math. Math. Sci. 27 (2004), 1429-1436.

https://doi.org/10.1155/S0161171204108090

[3] A. A. Attiya and M. F. Yassen, Some subordination and superordination results associated with generalized Srivastava-Attiya operator, Filomat 31(1) (2017), 53-60. https://doi.org/10.2298/FIL1701053A 
[4] S. D. Bernardi, Convex and starlike univalent functions, Trans. Amer. Math. Soc. 135 (1969), 429-446. https://doi.org/10.1090/S0002-9947-1969-0232920-2

[5] T. Bulboacă, Classes of first order differential superordinations, Demonstratio Math. 35(2) (2002), 287-292. https://doi.org/10.1515/dema-2002-0209

[6] N. E. Cho and H. M. Srivastava, Argument estimates of certain analytic functions defined by a class of multiplier transformations, Math. Comput. Modelling 37(1-2) (2003), 39-49. https://doi.org/10.1016/S0895-7177(03)80004-3

[7] N. E. Cho and T. H. Kim, Multiplier transformations and strongly close-to-convex functions, Bull. Korean Math. Soc. 40(3) (2003), 399-410. https://doi.org/10.4134/BKMS.2003.40.3.399

[8] I. B. Jung, Y. C. Kim, H. M. Srivastava, The Hardy space of analytic functions associated with certain one-parameter families of integral operators, J. Math. Anal. Appl. 176 (1993), 138-147. https://doi.org/10.1006/jmaa.1993.1204

[9] S. S. Miller and P. T. Mocanu, Differential Subordinations: Theory and Applications, Series on Monographs and Textbooks in Pure and Applied Mathematics, Vol. 225, Marcel Dekker Inc., New York and Basel, 2000. https://doi.org/10.1201/9781482289817

[10] S. Rahrovi, Subordination and superordination properties for convolution operator, Int. J. Nonlinear Anal. Appl. 6(2) (2015), 137-147.

[11] G. S. Salagean, Subclasses of univalent functions, Lecture Notes in Math., Vol. 1013, Springer Verlag, Berlin, 1983, pp. 362-372. https://doi.org/10.1007/BFb0066543

[12] T. M. Seoudy, Subordination and superordination results of $p$-valent analytic functions involving a linear operator, Bol. Soc. Parana. Mat. 35(2) (2017), 223-234. https://doi.org/10.5269/bspm.v35i2.21993

[13] H. M. Srivastava and A. A. Attiya, An integral operator associated with the HurwitzLerch zeta function and differential subordination, Integral Transforms Spec. Funct. 18(3) (2007), 207-216. https://doi.org/10.1080/10652460701208577

[14] S. R. Swamy, Inclusion properties of certain subclasses of analytic functions, Int. Math. Forum 7(36) (2012), 1751-1760.

[15] B. A. Uralegaddi and C. Somanatha, Certain classes of univalent functions, in: Current Topics in Analytic Function Theory, H. M. Srivastava and S. Own, eds., 371-374, World Scientific, Singapore, 1992. https://doi.org/10.1142/9789814355896_0032

[16] A. K. Wanas, New differential operator for holomorphic functions, Earthline J. Math. Sci. 2(2) (2019), 527-537. https://doi.org/10.34198/ejms.2219.527537 
[17] A. K. Wanas and A. S. Joudah, Sandwich theorems for certain subclasses of analytic functions defined by convolution structure with generalized operator, An. Univ. Oradea Fasc. Mat. XXI(1) (2014), 183-190.

[18] A. K. Wanas and A. A. Lupas, Sandwich theorems for multivalent analytic functions associated with differential operator, Kragujevac Journal of Mathematics 44(1) (2020), 27-39.

[19] A. K. Wanas and G. Murugusundaramoorthy, Differential sandwich results for Wanas operator of analytic functions, Mathematica Moravica 24(1) (2020), 17-28. 